\title{
Construction Of Good Rank-1 Lattice Rules Based On The Weighted Star Discrepancy
}

Stephen Joe

Department of Mathematics, University of Waikato, Private Bag 3105, Hamilton, New Zealand

E-mail: stephenj@math.waikato.ac.nz

Summary. The 'goodness' of a set of quadrature points in $[0,1]^{d}$ may be measured by the weighted star discrepancy. If the weights for the weighted star discrepancy are summable, then we show that for $n$ prime there exist $n$-point rank- 1 lattice rules whose weighted star discrepancy is $O\left(n^{-1+\delta}\right)$ for any $\delta>0$, where the implied constant depends on $\delta$ and the weights, but is independent of $d$ and $n$. Further, we show that the generating vector $\mathbf{z}$ for such lattice rules may be obtained using a component-by-component construction. The results given here for the weighted star discrepancy are used to derive corresponding results for a weighted $L_{p}$ discrepancy.

\section{Introduction}

Integrals over the $d$-dimensional unit cube given by

$$
I_{d}(f)=\int_{[0,1]^{d}} f(\mathbf{x}) \mathrm{d} \mathbf{x}
$$

may be approximated using $n$-point rank-1 lattice rules. These are quadrature rules of the form

$$
Q_{n, d}(f)=\frac{1}{n} \sum_{k=0}^{n-1} f\left(\left\{\frac{k \mathbf{z}}{n}\right\}\right),
$$

where $\mathbf{z} \in \mathbb{Z}^{d}$ is the 'generating vector' with no factor in common with $n$, and the braces around a vector indicate that we take the fractional part of each component of the vector. For our purposes, it is convenient to assume that $\operatorname{gcd}\left(z_{j}, n\right)=1$ for $1 \leq j \leq d$, where $z_{j}$ is the $j$-th component of $\mathbf{z}$.

The star discrepancy of the point set $P_{n}(\mathbf{z}):=\{\{k \mathbf{z} / n\}, 0 \leq k \leq n-1\}$ is defined by

$$
D^{*}\left(P_{n}(\mathbf{z})\right)=D_{n}^{*}(\mathbf{z}):=\sup _{\mathbf{x} \in[0,1)^{d}}\left|\operatorname{discr}\left(\mathbf{x}, P_{n}\right)\right|,
$$

where $\operatorname{discr}\left(\mathbf{x}, P_{n}\right)$ is the 'local discrepancy' defined by 


$$
\operatorname{discr}\left(\mathbf{x}, P_{n}\right):=\frac{\left|P_{n}(\mathbf{z}) \cap[\mathbf{0}, \mathbf{x})\right|}{n}-\operatorname{Vol}([\mathbf{0}, \mathbf{x})) .
$$

The star discrepancy occurs in the well-known Koksma-Hlawka inequality. Further details may be found in [3] and [19] or in more general works such as [11].

It is known (see [10] or [11]) that there exist $d$-dimensional rank-1 lattice rules whose star discrepancy is $O\left(n^{-1}(\ln (n))^{d}\right)$ with the implied constant depending on only $d$. For $n$ prime it was shown in [4] that such rules may be obtained by constructing their generating vectors component-by-component. In this paper we extend these results to the case of a weighted star discrepancy.

Such component-by-component constructions first appeared in [16], but there the integrands were in a periodic setting. Since then, there has been much work done in the $L_{2}$ case both in the periodic setting of weighted Korobov spaces and in the non-periodic setting of weighted Sobolev spaces (for example, see [7], [8], [9], [14], and [15]). Here we consider the weighted star discrepancy, since, as we shall see later, we are able to derive corresponding results for the weighted $L_{p}$ discrepancy.

In order to introduce the weighted star discrepancy, let $\mathfrak{u}$ be any subset of $\mathcal{D}:=\{1,2, \ldots, d-1, d\}$ with cardinality $|\mathfrak{u}|$. For the vector $\mathbf{x} \in[0,1]^{d}$, let $\mathbf{x}_{\mathfrak{u}}$ denote the vector from $[0,1]^{|\mathfrak{u}|}$ containing the components of $\mathbf{x}$ whose indices belong to $\mathfrak{u}$. By $\left(\mathbf{x}_{\mathfrak{u}}, \mathbf{1}\right)$ we mean the vector from $[0,1]^{d}$ whose $j$-th component is $x_{j}$ if $j \in \mathfrak{u}$ and 1 if $j \notin \mathfrak{u}$. From Zaremba's identity (see [17] or [19]) we have

$$
Q_{n, d}(f)-I_{d}(f)=\sum_{\emptyset \neq \mathfrak{u} \subseteq \mathcal{D}}(-1)^{|\mathfrak{u}|} \int_{[0,1]^{|\mathfrak{u}|}} \operatorname{discr}\left(\left(\mathbf{x}_{\mathfrak{u}}, \mathbf{1}\right), P_{n}\right) \frac{\partial^{|\mathfrak{u}|}}{\partial \mathbf{x}_{\mathfrak{u}}} f\left(\mathbf{x}_{\mathfrak{u}}, \mathbf{1}\right) \mathrm{d} \mathbf{x}_{\mathfrak{u}}
$$

Now let us introduce a sequence of positive weights $\left\{\gamma_{j}\right\}_{j=1}^{\infty}$ and set

$$
\gamma_{\mathfrak{u}}=\prod_{j \in \mathfrak{u}} \gamma_{j} \quad \text { with } \quad \gamma_{\emptyset}:=1
$$

Then we can write

$$
\begin{aligned}
& Q_{n, d}(f)-I_{d}(f) \\
= & \sum_{\emptyset \neq \mathfrak{u} \subseteq \mathcal{D}}(-1)^{|\mathfrak{u}|} \gamma_{\mathfrak{u}} \int_{[0,1]^{|\mathfrak{u}|}} \operatorname{discr}\left(\left(\mathbf{x}_{\mathfrak{u}}, \mathbf{1}\right), P_{n}\right) \boldsymbol{\gamma}_{\mathfrak{u}}^{-1} \frac{\partial^{|\mathfrak{u}|}}{\partial \mathbf{x}_{\mathfrak{u}}} f\left(\mathbf{x}_{\mathfrak{u}}, \mathbf{1}\right) \mathrm{d} \mathbf{x}_{\mathfrak{u}} .
\end{aligned}
$$

Applying Hölder's inequality for integrals and sums we obtain

$$
\begin{aligned}
\left|Q_{n, d}(f)-I_{d}(f)\right| \leq & \left(\sup _{\emptyset \neq \mathfrak{u} \subseteq \mathcal{D}} \sup _{\mathbf{x}_{\mathfrak{u}} \in[0,1]^{|u|}} \gamma_{\mathfrak{u}}\left|\operatorname{discr}\left(\left(\mathbf{x}_{\mathfrak{u}}, \mathbf{1}\right), P_{n}\right)\right|\right) \\
& \times\left(\sum_{\mathfrak{u} \subseteq \mathcal{D}} \gamma_{\mathfrak{u}}^{-1} \int_{[0,1]^{|\mathfrak{u}|}}\left|\frac{\partial^{|\mathfrak{u}|}}{\partial \mathbf{x}_{\mathfrak{u}}} f\left(\mathbf{x}_{\mathfrak{u}}, \mathbf{1}\right)\right| \mathrm{d} \mathbf{x}_{\mathfrak{u}}\right) .
\end{aligned}
$$


Then we can define a weighted star discrepancy $D_{n, \boldsymbol{\gamma}}^{*}(\mathbf{z})$ by

$$
D_{n, \gamma}^{*}(\mathbf{z}):=\sup _{\emptyset \neq \mathfrak{u} \subseteq \mathcal{D}} \gamma_{\mathfrak{u}} \sup _{\mathbf{x}_{\mathfrak{u}} \in[0,1]^{|u|}}\left|\operatorname{discr}\left(\left(\mathbf{x}_{\mathfrak{u}}, \mathbf{1}\right), P_{n}\right)\right| .
$$

In Section 2 we use an averaging argument to show that if the weights $\gamma_{j}$ are summable, there exist rank-1 lattice rules whose weighted star discrepancy is $O\left(n^{-1+\delta}\right)$ for any $\delta>0$, where the implied constant depends on $\delta$ and the weights. A more specific averaging argument is applied to lattice rules of the Korobov form, namely those for which $\mathbf{z}=\left(1, a, \ldots, a^{d-1}\right)(\bmod n)$, $1 \leq a \leq n-1$, to show there exist lattice rules of the Korobov form having $O\left(n^{-1+\delta}\right)$ weighted star discrepancy.

Besides existence results we are interested in how to find such lattice rules. One way, of course, is to find an appropriate $a$ in the Korobov form. However, such rules are not extensible in dimension; a value of $a$ that is good for one value of the dimension $d$ may not be good for a different value of the dimension. In Section 3 we present results showing that, alternatively, the generating vectors $\mathbf{z}$ for such lattice rules may be constructed a component at a time resulting in a $\mathbf{z}$ which is extensible in dimension. The cost of this componentby-component construction is $O\left(n^{2} d^{2}\right)$ operations, but it may be reduced to $O\left(n^{2} d\right)$ operations at the extra cost of $O(n)$ storage. It may be reduced even further to $O(n \ln (n) d)$ operations by making use of the approach proposed by Nuyens and Cools in [12]. We remark that constructions for polynomial lattice rules having small weighted star discrepancy have recently been proposed in [1]. As here, they consider a Korobov construction and a component-bycomponent construction.

The weighted star discrepancy considered here may be viewed as the $L_{\infty}$ version of a weighted $L_{p}$ discrepancy for $p \geq 1$. Weighted $L_{p}$ discrepancies have been considered in works such as [2] and [17]. In Section 4 we use the results obtained in Sections 2 and 3 for the weighted star discrepancy to derive corresponding results for the weighted $L_{p}$ discrepancy. Unlike the earlier results in the $L_{2}$ setting, the results presented here do not require the lattice points to be shifted.

\section{Rank-1 Lattice Rules Having Certain Weighted Star Discrepancy Bounds}

It follows from (4) that the weighted star discrepancy satisfies

$$
D_{n, \boldsymbol{\gamma}}^{*}(\mathbf{z}) \leq \sum_{\mathfrak{u} \subseteq \mathcal{D}} \gamma_{\mathfrak{u}} \sup _{\mathbf{x}_{\mathfrak{u}} \in[0,1]^{|u|}}\left|\operatorname{discr}\left(\left(\mathbf{x}_{\mathfrak{u}}, \mathbf{1}\right), P_{n}\right)\right|
$$

Moreover, it follows from [11, Theorem 3.10 and Theorem 5.6] (see also [2]) that

$$
\sup _{\mathbf{x}_{\mathfrak{u}} \in[0,1]^{|u|}}\left|\operatorname{discr}\left(\left(\mathbf{x}_{\mathfrak{u}}, \mathbf{1}\right), P_{n}\right)\right| \leq 1-(1-1 / n)^{|\mathfrak{u}|}+\frac{R_{n}(\mathbf{z}, \mathfrak{u})}{2},
$$


where

$$
R_{n}(\mathbf{z}, \mathfrak{u})=\sum_{\substack{\mathbf{h} \cdot \mathbf{z}_{\mathfrak{u}} \equiv 0\left(\bmod \\ \mathbf{h} \in C_{n,|\mathfrak{u}|}^{*}\right.}} \prod_{j=1}^{|\mathfrak{u}|} \frac{1}{\max \left(1,\left|h_{j}\right|\right)} .
$$

Here $\mathbf{z}_{\mathfrak{u}}$ is the vector consisting of the components of $\mathbf{z}$ whose indices belong to $\mathfrak{u}$ and

$$
C_{n,|\mathfrak{u}|}^{*}=\left\{\mathbf{h} \in \mathbb{Z}^{|\mathfrak{u}|}, \mathbf{h} \neq \mathbf{0}:-n / 2<h_{j} \leq n / 2,1 \leq j \leq|\mathfrak{u}|\right\} .
$$

We then obtain

$$
D_{n, \boldsymbol{\gamma}}^{*}(\mathbf{z}) \leq \sum_{\mathfrak{u} \subseteq \mathcal{D}} \gamma_{\mathfrak{u}}\left(1-(1-1 / n)^{|\mathfrak{u}|}+\frac{R_{n}(\mathbf{z}, \mathfrak{u})}{2}\right) .
$$

Under the assumption that $\operatorname{gcd}\left(z_{j}, n\right)=1$ for $1 \leq j \leq d$, then $\mathbf{z}_{\mathfrak{u}}$ is the generating vector for a $|\mathfrak{u}|$-dimensional rank-1 lattice rule having $n$ points. It then follows from the error theory of lattice rules (for example, see [11, Chapter 5] or [13, Chapter 4]) that we may write $R_{n}(\mathbf{z}, \mathfrak{u})$ as

$$
R_{n}(\mathbf{z}, \mathfrak{u})=\frac{1}{n} \sum_{k=0}^{n-1} \prod_{j \in \mathfrak{u}}\left(1+\sum_{-n / 2<h \leq n / 2}^{\prime} \frac{e^{2 \pi \mathrm{i} h k z_{j} / n}}{|h|}\right)-1
$$

where the' ${ }^{\prime}$ on the sum indicates that we omit the $h=0$ term.

Bounds on the weighted star discrepancy $D_{n, \boldsymbol{\gamma}}^{*}(\mathbf{z})$ may be obtained by making use of $(6)$. We first consider $\sum_{\mathfrak{u} \subseteq \mathcal{D}} \gamma_{\mathfrak{u}}\left(1-(1-1 / n)^{|\mathfrak{u}|}\right)$.

Lemma 1. Suppose the weights $\gamma_{j}$ are summable, that $i s, \sum_{j=1}^{\infty} \gamma_{j}<\infty$. Then

$$
\sum_{\mathfrak{u} \subseteq \mathcal{D}} \gamma_{\mathfrak{u}}\left(1-(1-1 / n)^{|\mathfrak{u}|}\right) \leq \frac{\max (1, \Gamma)}{n} \prod_{j=1}^{\infty}\left(1+\gamma_{j}\right) \leq \frac{\max (1, \Gamma) e^{\sum_{j=1}^{\infty} \gamma_{j}}}{n}
$$

where $\Gamma:=\sum_{j=1}^{\infty} \gamma_{j} /\left(1+\gamma_{j}\right)<\infty$

Proof. We may write

$$
\begin{aligned}
\sum_{\mathfrak{u} \subseteq \mathcal{D}} \gamma_{\mathfrak{u}}\left(1-(1-1 / n)^{|\mathfrak{u}|}\right) & =\prod_{j=1}^{d}\left(1+\gamma_{j}\right)-\prod_{j=1}^{d}\left(1+\gamma_{j}(1-1 / n)\right) \\
& =\prod_{j=1}^{d}\left(1+\gamma_{j}\right)\left[1-\prod_{j=1}^{d}\left(1-\frac{\gamma_{j}}{n\left(1+\gamma_{j}\right)}\right)\right] .
\end{aligned}
$$


According to [2] we have

$$
\ln \left(\prod_{j=1}^{d}\left(1-\frac{\gamma_{j}}{n\left(1+\gamma_{j}\right)}\right)\right) \geq \ln (1-1 / n) \sum_{j=1}^{d} \frac{\gamma_{j}}{1+\gamma_{j}}
$$

which leads to

$$
\sum_{\mathfrak{u} \subseteq \mathcal{D}} \gamma_{\mathfrak{u}}\left(1-(1-1 / n)^{|\mathfrak{u}|}\right) \leq \prod_{j=1}^{d}\left(1+\gamma_{j}\right)\left[1-\left(1-\frac{1}{n}\right)^{\sum_{j=1}^{d} \gamma_{j} /\left(1+\gamma_{j}\right)}\right]
$$

Since $0<\gamma_{j} /\left(1+\gamma_{j}\right)<\gamma_{j}$, we see that since the $\gamma_{j}$ are summable, then so are the $\gamma_{j} /\left(1+\gamma_{j}\right)$, that is, $\Gamma<\infty$.

If $\Gamma \leq 1$, then we have $(1-1 / n)^{\Gamma} \geq 1-1 / n$ and hence

$$
1-\left(1-\frac{1}{n}\right)^{\Gamma} \leq \frac{1}{n}
$$

Now suppose $\Gamma>1$ and set $v(x)=(1+x)^{\Gamma}-\Gamma x-1$ for $x>-1$. Then it is easily verified that $v^{\prime}(0)=0$. Moreover, $v^{\prime \prime}(0)=\Gamma^{2}-\Gamma$ which is positive for $\Gamma>1$. Since $v^{\prime}(x)<0$ for $-1<x<0$ and $v^{\prime}(x)>0$ for $x>0$, we deduce that if $\Gamma>1$, then $v(x) \geq v(0)=0$ or $(1+x)^{\Gamma} \geq \Gamma x+1$ for $x>-1$. With $x=-1 / n$ we thus obtain

$$
\left(1-\frac{1}{n}\right)^{\Gamma} \geq-\frac{\Gamma}{n}+1 \text { and so } 1-\left(1-\frac{1}{n}\right)^{\Gamma} \leq \frac{\Gamma}{n} .
$$

It then follows from (8) that

$$
\begin{aligned}
\sum_{\mathfrak{u} \subseteq \mathcal{D}} \gamma_{\mathfrak{u}}\left(1-(1-1 / n)^{|\mathfrak{u}|}\right) & \leq \prod_{j=1}^{d}\left(1+\gamma_{j}\right)\left[1-\left(1-\frac{1}{n}\right)^{\Gamma}\right] \\
& \leq \frac{\max (1, \Gamma)}{n} \prod_{j=1}^{d}\left(1+\gamma_{j}\right) \leq \frac{\max (1, \Gamma)}{n} \prod_{j=1}^{\infty}\left(1+\gamma_{j}\right) \\
& =\frac{\max (1, \Gamma)}{n} e^{\sum_{j=1}^{\infty} \ln \left(1+\gamma_{j}\right)} \leq \frac{\max (1, \Gamma) e^{\sum_{j=1}^{\infty} \gamma_{j}}}{n},
\end{aligned}
$$

where we have used $\ln (1+x) \leq x$ for $x \geq 0$.

With $\gamma_{\emptyset}=1$, we make use of (3) and (7) to next consider

$$
\begin{aligned}
R_{n, \boldsymbol{\gamma}}(\mathbf{z}) & :=\sum_{\mathfrak{u} \subseteq \mathcal{D}} \gamma_{\mathfrak{u}} R_{n}(\mathbf{z}, \mathfrak{u}) \\
& =\sum_{\mathfrak{u} \subseteq \mathcal{D}} \gamma_{\mathfrak{u}}\left[\frac{1}{n} \sum_{k=0}^{n-1} \prod_{j \in \mathfrak{u}}\left(1+\sum_{-n / 2<h \leq n / 2}^{\prime} \frac{e^{2 \pi \mathrm{i} h k z_{j} / n}}{|h|}\right)-1\right]
\end{aligned}
$$




$$
\begin{aligned}
& =\sum_{\mathfrak{u} \subseteq \mathcal{D}}\left[\frac{1}{n} \sum_{k=0}^{n-1} \prod_{j \in \mathfrak{u}} \gamma_{j}\left(1+\sum_{-n / 2<h \leq n / 2}^{\prime} \frac{e^{2 \pi \mathrm{i} h k z_{j} / n}}{|h|}\right)-\prod_{j \in \mathfrak{u}} \gamma_{j}\right] \\
& =\sum_{\mathfrak{u} \subseteq \mathcal{D}} \frac{1}{n} \sum_{k=0}^{n-1} \prod_{j \in \mathfrak{u}} \gamma_{j}\left(1+\sum_{-n / 2<h \leq n / 2}^{\prime} \frac{e^{2 \pi \mathrm{i} h k z_{j} / n}}{|h|}\right)-\prod_{j=1}^{d}\left(1+\gamma_{j}\right) .
\end{aligned}
$$

By interchanging the first two sums, we obtain

$$
\begin{aligned}
R_{n, \boldsymbol{\gamma}}(\mathbf{z}) & =\frac{1}{n} \sum_{k=0}^{n-1} \sum_{\mathfrak{u} \subseteq \mathcal{D}} \prod_{j \in \mathfrak{u}} \gamma_{j}\left(1+\sum_{-n / 2<h \leq n / 2}^{\prime} \frac{e^{2 \pi \mathrm{i} h k z_{j} / n}}{|h|}\right)-\prod_{j=1}^{d}\left(1+\gamma_{j}\right) \\
& =\frac{1}{n} \sum_{k=0}^{n-1} \prod_{j=1}^{d}\left(1+\gamma_{j}+\gamma_{j} \sum_{-n / 2<h \leq n / 2}^{\prime} \frac{e^{2 \pi \mathrm{i} h k z_{j} / n}}{|h|}\right)-\prod_{j=1}^{d}\left(1+\gamma_{j}\right) .
\end{aligned}
$$

Setting $\beta_{j}=1+\gamma_{j}$, we then see that

$$
R_{n, \boldsymbol{\gamma}}(\mathbf{z})=\frac{1}{n} \sum_{k=0}^{n-1} \prod_{j=1}^{d}\left(\beta_{j}+\gamma_{j} \sum_{-n / 2<h \leq n / 2}^{\prime} \frac{e^{2 \pi \mathrm{i} h k z_{j} / n}}{|h|}\right)-\prod_{j=1}^{d} \beta_{j} .
$$

In the case $d=1$, it is not hard to verify that $R_{n, \gamma}(\mathbf{z})=0$. We also see from this expression that for given dimension $d$, calculation of $R_{n, \boldsymbol{\gamma}}(\mathbf{z})$ would require $O\left(n^{2} d\right)$ operations. However, the asymptotic expansion techniques found in [5] may be used to reduce this to $O(n d)$ operations. Further details may be found in Appendix A.

We shall obtain bounds on $R_{n, \boldsymbol{\gamma}}(\mathbf{z})$ for the case in which $n$ is prime by obtaining an expression for the mean value of $R_{n, \boldsymbol{\gamma}}(\mathbf{z})$ taken over all integer vectors $\mathbf{z} \in \mathcal{Z}_{n}^{d}$, where $\mathcal{Z}_{n}=\{1,2, \ldots, n-1\}$. Thus the mean $M_{n, d, \boldsymbol{\gamma}}$ is defined by

$$
M_{n, d, \boldsymbol{\gamma}}:=\frac{1}{(n-1)^{d}} \sum_{\mathbf{z} \in \mathcal{Z}_{n}^{d}} R_{n, \boldsymbol{\gamma}}(\mathbf{z}) .
$$

Theorem 1. Let $n$ be a prime number. Then

$$
M_{n, d, \boldsymbol{\gamma}}=\frac{1}{n} \prod_{j=1}^{d}\left(\beta_{j}+\gamma_{j} S_{n}\right)+\frac{n-1}{n} \prod_{j=1}^{d}\left(\beta_{j}-\gamma_{j} \frac{S_{n}}{n-1}\right)-\prod_{j=1}^{d} \beta_{j}
$$

where

$$
S_{n}=\sum_{-n / 2<h \leq n / 2}^{\prime} \frac{1}{|h|}
$$

Proof. In (9) we can take out the $k=0$ term which is independent of $\mathbf{z}$ to obtain 


$$
\begin{aligned}
& M_{n, d, \gamma} \\
= & \frac{1}{n} \prod_{j=1}^{d}\left(\beta_{j}+\gamma_{j} S_{n}\right) \\
& +\frac{1}{n} \sum_{k=1}^{n-1} \prod_{j=1}^{d}\left[\frac{1}{n-1} \sum_{z_{j}=1}^{n-1}\left(\beta_{j}+\gamma_{j} \sum_{-n / 2<h \leq n / 2}^{\prime} \frac{e^{2 \pi \mathrm{i} h k z_{j} / n}}{|h|}\right)\right]-\prod_{j=1}^{d} \beta_{j} .
\end{aligned}
$$

Now define

$$
T_{n}(k):=\frac{1}{n-1} \sum_{z=1}^{n-1} \sum_{-n / 2<h \leq n / 2}^{\prime} \frac{e^{2 \pi \mathrm{i} h k z / n}}{|h|}, \quad 0 \leq k \leq n-1 .
$$

When $k=0, T_{n}(0)$ is simply $S_{n}$. For $n$ prime and $1 \leq k \leq n-1$ we see that $k$ cannot be a multiple of $n$, and nor can $h$ in the situation when $-n / 2<h \leq n / 2$ with $h \neq 0$. Hence $h k \not \equiv 0(\bmod n)$ and we have

$$
\begin{aligned}
T_{n}(k) & =\frac{1}{n-1} \sum_{-n / 2<h \leq n / 2}^{\prime} \sum_{z=1}^{n-1} \frac{e^{2 \pi \mathrm{i} h k z / n}}{|h|} \\
& =\frac{1}{n-1} \sum_{-n / 2<h \leq n / 2}^{\prime} \frac{1}{|h|}\left(\sum_{z=0}^{n-1}\left(e^{2 \pi \mathrm{i} h k / n}\right)^{z}-1\right)=\frac{-S_{n}}{n-1},
\end{aligned}
$$

which we note is independent of $k$. It then follows that

$$
M_{n, d, \gamma}=\frac{1}{n} \prod_{j=1}^{d}\left(\beta_{j}+\gamma_{j} S_{n}\right)+\frac{1}{n} \sum_{k=1}^{n-1} \prod_{j=1}^{d}\left(\beta_{j}+\gamma_{j} \frac{-S_{n}}{n-1}\right)-\prod_{j=1}^{d} \beta_{j},
$$

which leads to the desired result.

In the case $d=1$, the expression for $M_{n, 1, \gamma_{1}}$ simplifies to 0 , which is as expected, since for $d=1$ the values of $R_{n, \gamma_{1}}\left(z_{1}\right)$ are all zero.

Since $\beta_{j}=1+\gamma_{j}>\gamma_{j}$ and $S_{n} \leq n-1$, we have $\beta_{j}>\beta_{j}-\gamma_{j} S_{n} /(n-1) \geq 1$ and so

$$
\frac{n-1}{n} \prod_{j=1}^{d}\left(\beta_{j}-\gamma_{j} \frac{S_{n}}{n-1}\right)-\prod_{j=1}^{d} \beta_{j}<0 .
$$

Moreover, we have from [10, Lemmas 1 and 2] that $S_{n}<2 \ln (n)+1 / n^{2}-$ 0.2319 . So for $n \geq 3$ we have

$$
S_{n}<2 \ln (n)
$$

and direct calculation shows this holds for $n=2$ also. We then obtain the following corollary. 
Corollary 1. Let $n$ be a prime number. Then there exists a generating vector $\mathbf{z}$ such that

$$
R_{n, \boldsymbol{\gamma}}(\mathbf{z}) \leq \frac{1}{n} \prod_{j=1}^{d}\left(1+\gamma_{j}+\gamma_{j} S_{n}\right) \leq \frac{1}{n} \prod_{j=1}^{d}\left(1+\gamma_{j}+2 \gamma_{j} \ln (n)\right)
$$

Now recall from (6) and the definition of $R_{n, \boldsymbol{\gamma}}(\mathbf{z})$ that

$$
D_{n, \boldsymbol{\gamma}}^{*}(\mathbf{z}) \leq \sum_{\mathfrak{u} \subseteq \mathcal{D}} \gamma_{\mathfrak{u}}\left(1-(1-1 / n)^{|\mathfrak{u}|}\right)+\frac{R_{n, \boldsymbol{\gamma}}(\mathbf{z})}{2}
$$

This equation together with Lemma 1 and Corollary 1 show that if the $\gamma_{j}$ are summable, then there exists a generating vector $\mathbf{z}$ such that

$$
D_{n, \boldsymbol{\gamma}}^{*}(\mathbf{z}) \leq O\left(n^{-1}\right)+\frac{1}{2 n} \prod_{j=1}^{d}\left(1+\gamma_{j}+2 \gamma_{j} \ln (n)\right)
$$

where the implied constant depends on the weights, but is independent of $d$. This bound for $D_{n, \boldsymbol{\gamma}}^{*}(\mathbf{z})$ has a $\ln (n)$ dependency. In order to obtain a bound without this $\ln (n)$ dependency, we can make use of the next lemma (stated and proved in [2]) and conclude that there exists a generating vector $\mathbf{z}$ such that

$$
D_{n, \boldsymbol{\gamma}}^{*}(\mathbf{z})=O\left(n^{-1+\delta}\right),
$$

for any $\delta>0$, where the implied constant depends on $\delta$ and the weights, but is independent of $d$ and $n$.

Lemma 2. Let $\tilde{\gamma}_{j}=2 \gamma_{j} /\left(1+\gamma_{j}\right)$ and suppose that the $\gamma_{j}$ are summable so that

$$
\sum_{j=1}^{\infty} \tilde{\gamma}_{j}<\infty
$$

Then for any $\delta>0$, there exists $C(\tilde{\gamma}, \delta)$, independent of $d$ and $n$, such that

$$
\prod_{j=1}^{d}\left(1+\gamma_{j}+2 \gamma_{j} \ln (n)\right) \leq C(\tilde{\gamma}, \delta) n^{\delta} \prod_{j=1}^{\infty}\left(1+\gamma_{j}\right) \leq C(\tilde{\gamma}, \delta) n^{\delta} e^{\sum_{j=1}^{\infty} \gamma_{j}}
$$

We recall from Section 1 that lattice rules of the Korobov form are those for which $\mathbf{z}=\left(1, a, \ldots, a^{d-1}\right)(\bmod n)$ for some $a$ satisfying $1 \leq a \leq n-1$. Writing such generating vectors as $\mathbf{z}(a)$, we now define the mean

$$
\mu_{n, d, \boldsymbol{\gamma}}:=\frac{1}{n-1} \sum_{a=1}^{n-1} R_{n, \boldsymbol{\gamma}}(\mathbf{z}(a)) .
$$

The next result shows that $\mu_{n, d, \gamma}$ satisfies a bound of the same order as the one given in Corollary 1. Hence there exist lattice rules of the Korobov form which have $O\left(n^{-1+\delta}\right)$ weighted star discrepancy. 
Theorem 2. Let $n$ be a prime number. Then

$$
\mu_{n, d, \gamma} \leq \frac{d}{n-1} \prod_{j=1}^{d}\left(1+\gamma_{j}+\gamma_{j} S_{n}\right)
$$

Proof. The proof we present is similar to the proof of Theorem 1 in [18]. We see from (9) that $R_{n, \gamma}(\mathbf{z}(a))$ is the error from applying the lattice rule to the function

$$
f(\mathbf{x})=\sum_{\mathbf{h} \in C_{n, d}^{*} \cup\{\mathbf{0}\}} \frac{e^{2 \pi \mathrm{i} \mathbf{h} \cdot \mathbf{x}}}{\prod_{j=1}^{d} r\left(\gamma_{j}, h_{j}\right)},
$$

where

$$
r(\gamma, h)= \begin{cases}1+\gamma, & h=0 \\ |h| / \gamma, & h \neq 0\end{cases}
$$

It then follows from the theory of lattice rules that we may write

$$
R_{n, \boldsymbol{\gamma}}(\mathbf{z}(a))=\sum_{\mathbf{h} \in C_{n, d}^{*}} \frac{\delta_{n}(\mathbf{h} \cdot \mathbf{z}(a))}{\prod_{j=1}^{d} r\left(\gamma_{j}, h_{j}\right)},
$$

where $\delta_{n}(m)$ denotes one or zero depending on whether $m \equiv 0(\bmod n)$ or not.

From the definition of $\mu_{n, d, \boldsymbol{\gamma}}$, it follows that we have

$$
\mu_{n, d, \boldsymbol{\gamma}}=\frac{1}{n-1} \sum_{\mathbf{h} \in C_{n, d}^{*}} \prod_{j=1}^{d} \frac{1}{r\left(\gamma_{j}, h_{j}\right)} \sum_{a=1}^{n-1} \delta_{n}(\mathbf{h} \cdot \mathbf{z}(a)) .
$$

Since $\mathbf{h} \cdot \mathbf{z}(a)=h_{1}+h_{2} a+\cdots+h_{d} a^{d-1}$, we see this last sum is just the number of solutions of the congruence $h_{1}+h_{2} a+\cdots+h_{d} a^{d-1} \equiv 0(\bmod n)$. Now because $n$ is prime and $\mathbf{h} \in C_{n, d}^{*}$, then the greatest common divisor of the numbers $h_{1}, h_{2}, \ldots, h_{d}$ cannot be a multiple of $n$. It then follows from a well-known result in number theory (for example, see [6]) that the last sum in (14) is bounded by $d-1$. We then have

$$
\begin{aligned}
\mu_{n, d, \boldsymbol{\gamma}} & \leq \frac{d}{n-1} \sum_{\mathbf{h} \in C_{n, d}^{*}} \prod_{j=1}^{d} \frac{1}{r\left(\gamma_{j}, h_{j}\right)} \\
& <\frac{d}{n-1} \prod_{j=1}^{d}\left(1+\gamma_{j}+\gamma_{j} \sum_{-n / 2<h \leq n / 2}^{\prime} \frac{1}{|h|}\right),
\end{aligned}
$$

which leads to the desired bound. 


\section{A Component-By-Component Construction}

We shall now prove that for $n$ prime we can construct $\mathbf{z}$ component-bycomponent such that

$$
R_{n, \boldsymbol{\gamma}}(\mathbf{z}) \leq \frac{1}{n-1} \prod_{j=1}^{d}\left(\beta_{j}+\gamma_{j} S_{n}\right)
$$

where we recall that $\beta_{j}=1+\gamma_{j}$.

Theorem 3. Let $n$ be a prime number. Suppose there exists a $\mathbf{z} \in \mathcal{Z}_{n}^{d}$ such that

$$
R_{n, \boldsymbol{\gamma}}(\mathbf{z}) \leq \frac{1}{n-1} \prod_{j=1}^{d}\left(\beta_{j}+\gamma_{j} S_{n}\right), \quad \text { where } S_{n}=\sum_{-n / 2<h \leq n / 2}^{\prime} \frac{1}{|h|} .
$$

Then there exists $z_{d+1} \in \mathcal{Z}_{n}$ such that

$$
R_{n, \boldsymbol{\gamma}}\left(\mathbf{z}, z_{d+1}\right) \leq \frac{1}{n-1} \prod_{j=1}^{d+1}\left(\beta_{j}+\gamma_{j} S_{n}\right)
$$

Such a $z_{d+1}$ can be found by minimizing $R_{n, \boldsymbol{\gamma}}\left(\mathbf{z}, z_{d+1}\right)$ over the set $\mathcal{Z}_{n}$.

Proof. For any $z_{d+1} \in \mathcal{Z}_{n}$ we have from (9) that

$$
\begin{aligned}
R_{n, \boldsymbol{\gamma}}\left(\mathbf{z}, z_{d+1}\right)=\beta_{d+1} R_{n, \boldsymbol{\gamma}}(\mathbf{z}) & \\
& +\frac{\gamma_{d+1}}{n} \sum_{k=0}^{n-1}\left[\prod_{j=1}^{d}\left(\beta_{j}+\gamma_{j} \sum_{-n / 2<h \leq n / 2}^{\prime} \frac{e^{2 \pi \mathrm{i} h k z_{j} / n}}{|h|}\right)\right] \\
& \times\left[\sum_{-n / 2<h \leq n / 2}^{\prime} \frac{e^{2 \pi \mathrm{i} h k z_{d+1} / n}}{|h|}\right] .
\end{aligned}
$$

Next we average over the possible $n-1$ values of $z_{d+1}$ in the last term to form

$$
\frac{1}{n-1} \sum_{z_{d+1}=1}^{n-1} \sum_{-n / 2<h \leq n / 2}^{\prime} \frac{e^{2 \pi \mathrm{i} h k z_{d+1} / n}}{|h|}, \quad 0 \leq k \leq n-1 .
$$

However, this is just the quantity $T_{n}(k)$ defined previously in (10).

It then follows from (15) by separating out the $k=0$ term that there exists a $z_{d+1} \in \mathcal{Z}_{n}$ such that 


$$
\begin{aligned}
R_{n, \boldsymbol{\gamma}}\left(\mathbf{z}, z_{d+1}\right) \leq & \beta_{d+1} R_{n, \boldsymbol{\gamma}}(\mathbf{z})+\frac{\gamma_{d+1}}{n} \prod_{j=1}^{d}\left(\beta_{j}+\gamma_{j} S_{n}\right) S_{n} \\
& +\frac{\gamma_{d+1}}{n} \sum_{k=1}^{n-1} \prod_{j=1}^{d}\left(\beta_{j}+\gamma_{j} \sum_{-n / 2<h \leq n / 2}^{\prime} \frac{e^{2 \pi \mathrm{i} h k z_{j} / n}}{|h|}\right) T_{n}(k) \\
= & \beta_{d+1} R_{n, \gamma}(\mathbf{z})+\frac{\gamma_{d+1}}{n} \prod_{j=1}^{d}\left(\beta_{j}+\gamma_{j} S_{n}\right) S_{n} \\
& +\frac{\gamma_{d+1}}{n} \sum_{k=1}^{n-1} \prod_{j=1}^{d}\left(\beta_{j}+\gamma_{j} \sum_{-n / 2<h \leq n / 2}^{\prime} \frac{e^{2 \pi \mathrm{i} h k z_{j} / n}}{|h|}\right)\left(\frac{-S_{n}}{n-1}\right) \\
= & \beta_{d+1} R_{n, \boldsymbol{\gamma}}(\mathbf{z})+\frac{\gamma_{d+1}}{n} \prod_{j=1}^{d}\left(\beta_{j}+\gamma_{j} S_{n}\right) S_{n} \\
& +\frac{\gamma_{d+1} S_{n}}{n-1}\left(-\frac{1}{n} \sum_{k=0}^{n-1} \prod_{j=1}^{d}\left(\beta_{j}+\gamma_{j} \sum_{-n / 2<h \leq n / 2}^{\prime}\right.\right. \\
& \left.+\frac{e^{2 \pi \mathrm{i} h k z_{j} / n}}{|h|}\right)
\end{aligned}
$$

where we have made use of (11) and in the last equation, subtracted and added in the $k=0$ term. By using (9) we find that for this $z_{d+1}$ we have

$$
\begin{aligned}
R_{n, \boldsymbol{\gamma}}\left(\mathbf{z}, z_{d+1}\right) \leq & \beta_{d+1} R_{n, \boldsymbol{\gamma}}(\mathbf{z})+\frac{\gamma_{d+1}}{n} \prod_{j=1}^{d}\left(\beta_{j}+\gamma_{j} S_{n}\right) S_{n} \\
& \quad+\frac{\gamma_{d+1} S_{n}}{n-1}\left(-R_{n, \boldsymbol{\gamma}}(\mathbf{z})-\prod_{j=1}^{d} \beta_{j}+\frac{1}{n} \prod_{j=1}^{d}\left(\beta_{j}+\gamma_{j} S_{n}\right)\right) \\
\leq & \beta_{d+1} R_{n, \boldsymbol{\gamma}}(\mathbf{z})+\frac{\gamma_{d+1} S_{n}}{n}\left(\prod_{j=1}^{d}\left(\beta_{j}+\gamma_{j} S_{n}\right)\right)\left(1+\frac{1}{n-1}\right) \\
= & \beta_{d+1} R_{n, \boldsymbol{\gamma}}(\mathbf{z})+\frac{\gamma_{d+1} S_{n}}{n-1} \prod_{j=1}^{d}\left(\beta_{j}+\gamma_{j} S_{n}\right) \\
\leq & \frac{1}{n-1}\left(\prod_{j=1}^{d}\left(\beta_{j}+\gamma_{j} S_{n}\right)\right)\left(\beta_{d+1}+\gamma_{d+1} S_{n}\right) \\
= & \frac{1}{n-1} \prod_{j=1}^{d+1}\left(\beta_{j}+\gamma_{j} S_{n}\right),
\end{aligned}
$$


where we have made use of the fact that $R_{n, \gamma}(\mathbf{z})$ satisfies the assumed bound. This completes the proof.

Recalling that for $d=1$ we have $R_{n, \gamma_{1}}\left(z_{1}\right)=0$, the previous theorem leads to the following corollary.

Corollary 2. Let $n$ be a prime number. We can construct $\mathbf{z} \in \mathcal{Z}_{n}^{d}$ componentby-component such that for all $s=1, \ldots, d$,

$$
R_{n, \boldsymbol{\gamma}}\left(z_{1}, \ldots, z_{s}\right) \leq \frac{1}{n-1} \prod_{j=1}^{s}\left(\beta_{j}+\gamma_{j} S_{n}\right)
$$

We can set $z_{1}=1$, and for $2 \leq s \leq d$, each $z_{s}$ can be found by minimizing $R_{n, \boldsymbol{\gamma}}\left(z_{1}, \ldots, z_{s}\right)$ over the set $\mathcal{Z}_{n}$.

Since $1 /(n-1) \leq 2 / n$ for $n \geq 2$, this corollary together with (12) and (13) show that for $n$ a prime number, we can construct $\mathbf{z}$ component-by-component such that

$$
D_{n, \gamma}^{*}(\mathbf{z}) \leq \sum_{\mathfrak{u} \subseteq \mathcal{D}} \gamma_{\mathfrak{u}}\left(1-(1-1 / n)^{|\mathfrak{u}|}\right)+\frac{1}{n} \prod_{j=1}^{d}\left(1+\gamma_{j}+2 \gamma_{j} \ln (n)\right) .
$$

If the $\gamma_{j}$ are summable we then see from Lemma 1 and Lemma 2 that the rank-1 lattice rule constructed in this manner is such that

$$
D_{n, \gamma}^{*}(\mathbf{z})=O\left(n^{-1+\delta}\right), \quad \delta>0,
$$

where the implied constant depends on $\delta$ and the weights, but is independent of $d$ and $n$.

Appendix A shows that $R_{n, \gamma}(\mathbf{z})$ may be calculated using asymptotic expansion techniques in $O(n d)$ operations. This together with Corollary 2 then shows that the cost of constructing the integer vector $\mathbf{z}$ up to dimension $d$ is $O\left(n^{2} d^{2}\right)$ operations. This can be reduced to $O\left(n^{2} d\right)$ operations if we store the products during the construction, but this would be at the expense of $O(n)$ storage. We remark that in [12], Nuyens and Cools proposed a more efficient implementation of the component-by-component construction. Their construction of $\mathbf{z}$ was based on minimizing a function of the form

$$
\frac{1}{n} \sum_{k=0}^{n-1} \prod_{j=1}^{d}\left(1+\gamma_{j} \omega\left(\left\{\frac{k z_{j}}{n}\right\}\right)\right)-1
$$

where $\omega$ was a certain function. Now we see from (9) that $R_{n, \boldsymbol{\gamma}}(\mathbf{z})$ may be written in a similar form since

$$
R_{n, \gamma}(\mathbf{z})=\frac{1}{n} \sum_{k=0}^{n-1} \prod_{j=1}^{d}\left(\beta_{j}+\gamma_{j} \omega\left(\left\{\frac{k z_{j}}{n}\right\}\right)\right)-\prod_{j=1}^{d} \beta_{j}
$$


where

$$
\omega(x)=\sum_{-n / 2<h \leq n / 2}^{\prime} \frac{e^{2 \pi \mathrm{i} h x}}{|h|}, \quad x \in[0,1] .
$$

With some minor modifications, the approach of Nuyens and Cools may then be used to similarly speed up the component-by-component construction proposed here so that only $O(n \ln (n) d)$ operations are required.

\section{Results For The Weighted $L_{p}$ Discrepancy}

In this section we apply the results of the previous two sections to obtain corresponding results for the weighted $L_{p}$ discrepancy which we define below. From Zaremba's identity given in (2) one can apply Hölder's inequality for integrals and sums to obtain

$$
\left|Q_{n, d}(f)-I_{d}(f)\right| \leq D_{n, \boldsymbol{\gamma}, p}(\mathbf{z})\left(\sum_{\mathfrak{u} \subseteq \mathcal{D}} \gamma_{\mathfrak{u}}^{-q} \int_{[0,1]^{|\mathfrak{u}|}}\left|\frac{\partial^{|\mathfrak{u}|}}{\partial \mathbf{x}_{\mathfrak{u}}} f\left(\mathbf{x}_{\mathfrak{u}}, \mathbf{1}\right)\right|^{q} \mathrm{~d} \mathbf{x}_{\mathfrak{u}}\right)^{1 / q}
$$

where $D_{n, \boldsymbol{\gamma}, p}(\mathbf{z})$, the weighted $L_{p}$ discrepancy, is defined by

$$
D_{n, \gamma, p}(\mathbf{z}):=\left(\sum_{\emptyset \neq \mathfrak{u} \subseteq \mathcal{D}} \gamma_{\mathfrak{u}}^{p} \int_{[0,1]|u|}\left|\operatorname{discr}\left(\left(\mathbf{x}_{\mathfrak{u}}, \mathbf{1}\right), P_{n}\right)\right|^{p} \mathrm{~d} \mathbf{x}_{\mathfrak{u}}\right)^{1 / p}
$$

with the local discrepancy discr $\left(\mathbf{x}, P_{n}\right)$ for any $\mathbf{x} \in[0,1]^{d}$ being defined in (1) and $1 / p+1 / q=1, p, q \geq 1$. Then we see that we have

$$
D_{n, \boldsymbol{\gamma}, p}(\mathbf{z}) \leq\left[\sum_{\mathfrak{u} \subseteq \mathcal{D}}\left(\gamma_{\mathfrak{u}} \sup _{\mathbf{x}_{\mathfrak{u}} \in[0,1]^{|\mathfrak{u}|}}\left|\operatorname{discr}\left(\left(\mathbf{x}_{\mathfrak{u}}, \mathbf{1}\right), P_{n}\right)\right|\right)^{p}\right]^{1 / p} .
$$

Now Jensen's inequality shows that for $\lambda \geq 1$,

$$
\left(\sum a_{i}^{\lambda}\right)^{1 / \lambda} \leq \sum a_{i}
$$

where the $a_{i}$ are arbitrary non-negative numbers. So for $p \geq 1$ we can take $\lambda=p$ and hence obtain

$$
D_{n, \boldsymbol{\gamma}, p}(\mathbf{z}) \leq \sum_{\mathfrak{u} \subseteq \mathcal{D}} \gamma_{\mathfrak{u}} \sup _{\mathbf{x} \in[0,1]^{|\mathfrak{u}|}}\left|\operatorname{discr}\left(\left(\mathbf{x}_{\mathfrak{u}}, \mathbf{1}\right), P_{n}\right)\right| .
$$

The bound on the right-hand side of this expression is the bound analyzed in Section 2 (for example, see (5) and (6)). Hence the results from that section and Section 3 hold. Suppose we apply the component-by-component algorithm 
implied in Corollary 2. Then, under the assumption that the weights are summable, the generating vector $\mathbf{z}$ constructed yields a point set that not only has a weighted star discrepancy of $O\left(n^{-1+\delta}\right), \delta>0$, but also has a weighted $L_{p}$ discrepancy of the same order.

In the case $p=2$, Kuo [7] showed that the component-by-component algorithm achieves the optimal $O\left(n^{-1+\delta}\right)$ rate for the weighted $L_{2}$ discrepancy if the sum of the square roots of the weights is finite. Since the weights used in [7] are the squares of the weights considered in this paper, the condition in [7] is equivalent to the condition here that the weights are summable. Moreover, the proof of the result in [7] was in a randomized setting, that is, it applied only to randomly shifted lattice rules. In contrast, the previous paragraph indicates that under the same condition on the weights, the component-bycomponent contruction presented here yields a purely deterministic point set whose weighted $L_{2}$ discrepancy is $O\left(n^{-1+\delta}\right)$.

\section{A Calculation of $\boldsymbol{R}_{n, \gamma}(\mathrm{z})$}

Here we provide details of how

$$
R_{n, \boldsymbol{\gamma}}(\mathbf{z})=\frac{1}{n} \sum_{k=0}^{n-1} \prod_{j=1}^{d}\left(\beta_{j}+\gamma_{j} \sum_{-n / 2<h \leq n / 2}^{\prime} \frac{e^{2 \pi \mathrm{i} h k z_{j} / n}}{|h|}\right)-\prod_{j=1}^{d} \beta_{j}
$$

may be calculated in $O(n d)$ operations. We see that because $\left\{k z_{j} / n\right\}=m / n$ for some $m$ satisfying $0 \leq m \leq n-1$, then to calculate $R_{n, \boldsymbol{\gamma}}(\mathbf{z})$ we need to have the values of

$$
\beta_{j}+\gamma_{j} \sum_{-n / 2<h \leq n / 2}^{\prime} \frac{e^{2 \pi \mathrm{i} h m / n}}{|h|}, \quad 0 \leq m \leq n-1 .
$$

However, if

$$
f_{n}(x)=1+\sum_{-n / 2<h \leq n / 2}^{\prime} \frac{e^{2 \pi \mathrm{i} h x}}{|h|}, \quad x \in[0,1]
$$

then

$$
\beta_{j}+\gamma_{j} \sum_{-n / 2<h \leq n / 2}^{\prime} \frac{e^{2 \pi \mathrm{i} h m / n}}{|h|}=\beta_{j}+\gamma_{j}\left(f_{n}(m / n)-1\right) .
$$

Since $\overline{f_{n}(1-x)}=f_{n}(x)$ for $x \in[0,1]$, then to calculate $R_{n, \boldsymbol{\gamma}}(\mathbf{z})$ we need to have the values of $f_{n}(m / n)$ for $0 \leq m \leq\lfloor n / 2\rfloor$. These $\lfloor n / 2\rfloor+1$ values may be calculated once and then stored.

Suppose we wish to calculate $f_{n}(m / n)$ with an absolute error of at most $\varepsilon$. Then the results in [5] show that if $\ell$ and $L$ are positive integers satisfying

$$
2 \leq \ell \leq\left(\frac{6 n^{2}}{\pi^{2}}\right)^{1 / 3} \quad \text { and } \quad \frac{4(L+1) !}{(\ell-1)^{L+2} \pi^{L+2}} \leq \varepsilon,
$$


then we should calculate $f_{n}(m / n)$ directly using (16) for $0 \leq m<\ell$. For $\ell \leq m \leq\lfloor n / 2\rfloor$ we use the approximation $G(m / n)$, where for $n$ odd,

$$
G(x)=1-2 \ln (2|\sin (\pi x)|)-2 \sum_{i=0}^{L} b_{i}(x) \cos (\pi[(n+i) x+(i+1) / 2]) .
$$

In this expression, $b_{0}(x)=1 /[(n+1)|\sin (\pi x)|]$ and

$$
b_{i+1}(x)=\frac{-(i+1)}{(n+2 i+3)|\sin (\pi x)|} b_{i}(x) .
$$

Similar expressions for $G$ and the $b_{i}$ are available when $n$ is an even number.

When $\varepsilon=2.0 \times 10^{-16}$, then for $n \geq 115$, (17) is satisfied with the choices $\ell=20$ and $L=14$. As another example, if $\varepsilon=1.0 \times 10^{-18}$, then for $n \geq 161$, we can take $\ell=25$ and $L=15$. So we see that the $\lfloor n / 2\rfloor+1$ values of $f_{n}(m / n)$ required may be obtained with an absolute error of at most $\varepsilon$ in $O(\ell n)+O(L) \times(\lfloor n / 2\rfloor+1-\ell)=O(n)$ operations which means that even if $n$ is large, $R_{n, \gamma}(\mathbf{z})$ may be calculated in $O(n d)$ operations.

\section{Acknowledgments}

The author would like to thank Henryk Woźniakowski for the suggestion to consider the weighted $L_{p}$ discrepancy case and for his detailed and constructive comments on an earlier version of this manuscript. These comments included the derivation of the bound given in Lemma 1. The author would also like to thank Greg Wasilkowski for bringing to his attention the result given in Lemma 2.

\section{References}

1. J. Dick, G. Leobacher, F. Pillichshammer, Construction algorithms for digital nets with small weighted star discrepancy, SIAM J. Numer. Anal., to appear.

2. F.J. Hickernell, H. Niederreiter, The existence of good extensible rank-1 lattices, J. Complexity 19 (2003) 286-300.

3. E. Hlawka, Funktionen von beschränkter Variation in der Theorie der Gleichverteilung, Ann. Mat. Pura ed Appl. 54 (1961) 325-334.

4. S. Joe, Component by component construction of rank-1 lattice rules having $O\left(n^{-1}(\ln (n))^{d}\right)$ star discrepancy, in: H. Niederreiter (Ed), Monte Carlo and Quasi-Monte Carlo Methods 2002, Springer, 2004, pp. 293-298.

5. S. Joe, I.H. Sloan, On computing the lattice rule criterion $R$, Math. Comp. 59 (1992) 557-568.

6. N.M. Korobov, Properties and calculation of optimal coefficients, Soviet Math. Dokl. 1 (1960) 696-700. 
7. F.Y. Kuo, Component-by-component constructions achieve the optimal rate of convergence for multivariate integration in weighted Korobov and Sobolev spaces, J. Complexity 19 (2003) 301-320.

8. F.Y. Kuo, S. Joe, Component-by-component construction of good lattice rules with a composite number of points, J. Complexity 18 (2002) 943-976.

9. F.Y. Kuo, S. Joe, Component-by-component construction of good intermediaterank lattice rules, SIAM J. Numer. Anal. 41 (2003) 1465-1486.

10. H. Niederreiter, Existence of good lattice points in the sense of Hlawka, Monatsh. Math. 86 (1978) 203-219.

11. H. Niederreiter, Random Number Generation and Quasi-Monte Carlo Methods, SIAM, Philadelphia, 1992.

12. D. Nuyens, R. Cools, Fast algorithms for component-by-component construction of rank-1 lattice rules in shift-invariant reproducing kernel Hilbert spaces, Math. Comp., to appear.

13. I.H. Sloan, S. Joe, Lattice Methods for Multiple Integration, Clarendon Press, Oxford, 1994

14. I.H. Sloan, F.Y. Kuo, S. Joe, On the step-by-step construction of quasi-Monte Carlo integration rules that achieve strong tractability error bounds in weighted Sobolev spaces, Math. Comp. 71 (2002) 1609-1640.

15. I.H. Sloan, F.Y. Kuo, S. Joe, Constructing randomly shifted lattice rules in weighted Sobolev spaces, SIAM J. Numer. Anal. 40 (2002) 1650-1665.

16. I.H. Sloan, A.V. Reztsov, Component-by-component construction of good lattice rules, Math. Comp. 71 (2002) 263-273.

17. I.H. Sloan, H. Woźniakowski, When are quasi-Monte Carlo algorithms efficient for high dimensional integrals?, J. Complexity 14 (1998) 1-33.

18. X. Wang, I.H. Sloan, J. Dick, On Korobov lattice rules in weighted spaces, SIAM J. Numer. Anal. 42 (2004) 1760-1779.

19. S.K. Zaremba, Some applications of multidimensional integration by parts, Ann. Polon. Math. 21 (1968) 85-96. 